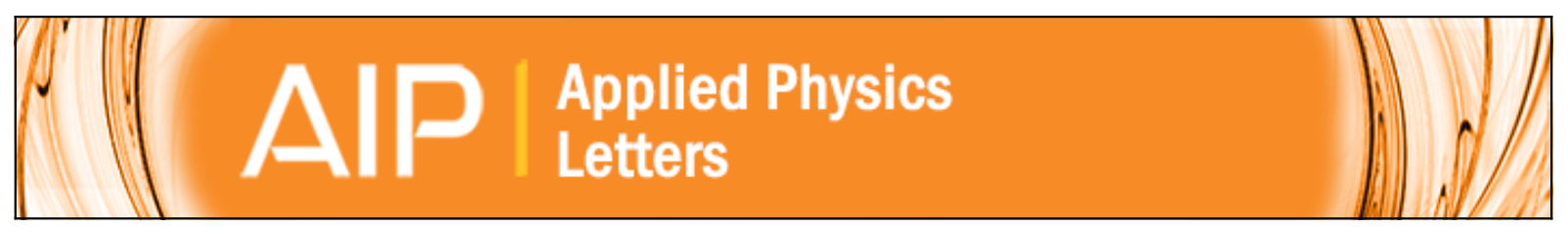

\title{
Role of intrinsic molecular dipole in energy level alignment at organic interfaces
}

Linda Lindell, Deniz Çakır, Geert Brocks, Mats Fahlman, and Slawomir Braun

Citation: Applied Physics Letters 102, 223301 (2013); doi: 10.1063/1.4809567

View online: http://dx.doi.org/10.1063/1.4809567

View Table of Contents: http://scitation.aip.org/content/aip/journal/apl/102/22?ver=pdfcov

Published by the AIP Publishing

\section{Articles you may be interested in}

Fermi level pinning by integer charge transfer at electrode-organic semiconductor interfaces

Appl. Phys. Lett. 98, 113303 (2011); 10.1063/1.3565963

Energy-level alignment and charge injection at metal/ C 60 /organic interfaces

Appl. Phys. Lett. 95, 043302 (2009); 10.1063/1.3189176

Energy level alignment at organic semiconductor/metal interfaces: Effect of polar self-assembled monolayers at the interface

J. Chem. Phys. 128, 074705 (2008); 10.1063/1.2832306

Energy-level alignment at interfaces between metals and the organic semiconductor $4,4^{\prime}-\mathrm{N}, \mathrm{N}^{\prime}$-dicarbazolylbiphenyl

J. Appl. Phys. 84, 3236 (1998); 10.1063/1.368477

Molecular level alignment at organic semiconductor-metal interfaces

Appl. Phys. Lett. 73, 662 (1998); 10.1063/1.121940

\section{AIP $\left.\right|_{\text {APL Photonics }}$}

APL Photonics is pleased to announce Benjamin Eggleton as its Editor-in-Chief

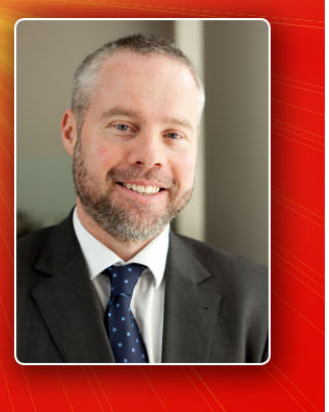




\title{
Role of intrinsic molecular dipole in energy level alignment at organic interfaces
}

\author{
Linda Lindell, ${ }^{1}$ Deniz Cakır, ${ }^{2}$ Geert Brocks, ${ }^{2}$ Mats Fahlman, ${ }^{1}$ and Slawomir Braun ${ }^{1}$ \\ ${ }^{1}$ Department of Physics, Chemistry and Biology, Linköping University, 58183 Linköping, Sweden \\ ${ }^{2}$ Computational Materials Science, Faculty of Science and Technology and MESA+Institute \\ for Nanotechnology, University of Twente, P.O. Box 217, 7500 AE Enschede, The Netherlands
}

(Received 12 October 2012; accepted 16 May 2013; published online 5 June 2013)

\begin{abstract}
The energy level alignment in metal-organic and organic-organic junctions of the widely used materials tris-(8-hydroxyquinoline)aluminum $\left(\mathrm{Alq}_{3}\right)$ and 1,4,5,8-naphthalenetetracarboxylic dianhydride (NTCDA) is investigated. The measured alignment schemes for single and bilayer films of $\mathrm{Alq}_{3}$ and NTCDA are interpreted with the integer charge transfer (ICT) model. Single layer films of $\mathrm{Alq}_{3}$ feature a constant vacuum level shift of $\sim 0.2-0.4 \mathrm{eV}$ in the absence of charge transfer across the interface. This finding is attributed to the intrinsic dipole of the $\mathrm{Alq}_{3}$ molecule and (partial) ordering of the molecules at the interfaces. The vacuum level shift changes the onset of Fermi level pinning, as it changes the energy needed for equilibrium charge transfer across the interface. (C) 2013 AIP Publishing LLC. [http://dx.doi.org/10.1063/1.4809567]
\end{abstract}

All organic-based electronic devices are made by deposition of successive layers (metal, oxide, insulating or semiconducting layers), and many key electronic processes (such as charge injection from metallic electrodes, charge recombination into light or light conversion into charges) occur at interfaces. In fact, as charges are localized on (parts of) molecules in most films, even charge transport can be seen as a sequence of charge injection events at organic-organic junctions. A device performance is thus strongly linked to the energy level alignment at the various interfaces contained within, and it is of great importance to understand and predict energy level alignment at both metal-organic and organic-organic interfaces.

Two different models are typically used to describe the energy level alignment at interfaces: the Induced Density of Interface States (IDIS) model $^{1}$ and the Integer Charge Transfer (ICT) model. ${ }^{2-4}$ According to the IDIS model, the energy level alignment at an organic-organic heterojunction does not depend on the underlying substrate, nor on the order in which the two organic layers are deposited on the substrate. In contrast, the ICT model gives an energy level alignment profile that depends on the underlying substrate and on the deposition order, provided that charge equilibration can take place over the whole system. ${ }^{4-7}$

The ICT model, used in this work, is designed for (hybrid) organic interfaces, where the interaction between the organic material and the substrate is weak. This can occur, for instance, for a metal substrate that is passivated by a native oxide layer, which will often be the case for metal electrodes applied in devices. Electrons can pass freely through this thin barrier in order to equilibrate the electrochemical potentials of the metal and the organic material. Experimentally, the potential step $\Delta$ at an interface is a very non-linear function of the substrate work function $\Phi_{\text {SUB }}$. An organic material can be characterized by two energy levels $\mathrm{E}_{\mathrm{ICT}-}$ and $\mathrm{E}_{\mathrm{ICT}+}$, where $\mathrm{E}_{\mathrm{ICT}-}<\mathrm{E}_{\mathrm{ICT}+}$. The interface potential step $\Delta$, depends on $\Phi_{\mathrm{SUB}}$ with respect to $\mathrm{E}_{\mathrm{ICT}+/ \text { - }}$, i.e., (i) $\Delta=\mathrm{E}_{\mathrm{ICT}-}-\Phi_{\mathrm{SUB}}$ for $\Phi_{\mathrm{SUB}}<\mathrm{E}_{\mathrm{ICT}-}$, (ii) $\Delta=0$ for $\mathrm{E}_{\mathrm{ICT}-}$ $<\Phi_{\mathrm{SUB}}<\mathrm{E}_{\mathrm{ICT}+}$, and (iii) $\Delta=\mathrm{E}_{\mathrm{ICT}+}-\Phi_{\mathrm{SUB}}$ for $\Phi_{\mathrm{SUB}}$
$>\mathrm{E}_{\mathrm{ICT}+} \cdot{ }^{2-4}$ In terms of the work function of the complete system $\Phi_{\mathrm{ORG} / \mathrm{SUB}}=\Phi_{\mathrm{SUB}}+\Delta$, these three regimes are characterized as, (i) $\Phi_{\mathrm{ORG} / \mathrm{SUB}}=\mathrm{E}_{\mathrm{ICT}-}$, (ii) $\Phi_{\mathrm{ORG} / \mathrm{SUB}}=\Phi_{\mathrm{SUB}}$, and (iii) $\Phi_{\mathrm{ORG} / \mathrm{SUB}}=\mathrm{E}_{\mathrm{ICT}+}$. Obviously, regime (ii) corresponds to the Schottky-Mott limit, or vacuum level alignment, where there is negligible electron transfer between the substrate and the organic material in equilibrium, whereas regimes (i) and (iii) represent pinning of the Fermi level by the organic material. The pinning levels $\mathrm{E}_{\mathrm{ICT}+}$ and $\mathrm{E}_{\mathrm{ICT}-}$ can be interpreted as the oxidation/reduction energies of the molecules at the interface, taking all electrostatic interactions at the interface into account. ${ }^{3,4,8,9}$ As stated, the ICT model relies on electron transfer across interfaces, and assuming that equilibration takes place across the whole multilayer, all layers can influence one another. Potential steps do not need to be localized at interfaces and depend explicitly on the order of the individual layers in the multilayer. This reasoning has been tested on the data of a number of experimental studies on donor/acceptor multilayers. ${ }^{5-7,10}$

In this paper, we study metal-organic junctions of tris(8-hydroxyquinoline)aluminum $\left(\mathrm{Alq}_{3}\right)$ and 1,4,5,8-naphthalenetetracarboxylic dianhydride (NTCDA) with a range of metals of different work functions, as well as the organicorganic $\mathrm{Alq}_{3}$-NTCDA heterojunction. Both these organic materials are frequently used in organic (opto)electronics. ${ }^{11}$ The $\mathrm{Alq}_{3}$ molecule has a large intrinsic dipole moment, ${ }^{12}$ making the energy level alignment at junctions with $\mathrm{Alq}_{3}$ more complex than that at previously studied junctions (where the molecules had a zero dipole moment). ${ }^{5-7,10}$ The work function of the samples is measured with an accuracy of $\pm 0.05 \mathrm{eV}$ by means of ultraviolet photoelectron spectroscopy (UPS) using monochromatized HeI radiation $(h \nu=21.2 \mathrm{eV})$ in a spectrometer of our own design and construction. The work function values are derived from the onset of the secondary electron cut-off. The substrate work functions used in the experiments range from $\sim 3.5 \mathrm{eV}$ for $\mathrm{AlO}_{\mathrm{x}}$ up to as high as $\sim 5.8$ for UV-ozone treated gold and poly(3,4-ethylenedioxythiophene)-poly(perfluoroethylene sulfonic acid) (PEDOT-PFESA). All of the substrates 
are handled under ambient conditions and thus feature the non-reactive surfaces required by the ICT model. ${ }^{3,4}$ Both NTCDA and $\mathrm{Alq}_{3}$ are deposited onto the substrates by insitu vacuum sublimation from a Knudsen-cell. NTCDA $(\geq 95.0 \%)$ and $\mathrm{Alq}_{3}(99.995 \%)$ were purchased from Fluka and Aldrich, respectively. The substrate temperature $\mathrm{T}_{\text {substrate }}$ is set to $20^{\circ} \mathrm{C}$ during deposition and measurements. The pinning energies of NTCDA and $\mathrm{Alq}_{3}$ are calculated using a model based upon density functional theory described in detail elsewhere. ${ }^{7,9,13}$

In Figure 1, the UPS-derived work functions after film formation of NTCDA (yellow) and $\mathrm{Alq}_{3}$ (teal) $\Phi_{\mathrm{ORG} / \mathrm{SUB}}$ are plotted versus the substrate work function $\Phi_{\text {SUB }}$. The nonlinear behavior suggests an analysis in terms of the ICT model. For NTCDA, the analysis of the energy level alignment is straightforward. From the zero slope part of the NTCDA plot in Figure 1, it is seen that the Fermi level is

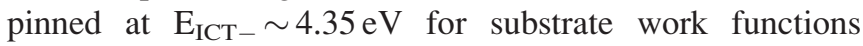

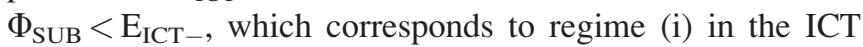
model as stated earlier. For substrate work functions $\Phi_{\text {SUB }}>\mathrm{E}_{\mathrm{ICT}-}, \Phi_{\mathrm{ORG} / \mathrm{SUB}}$ follows the Schottky-Mott limit of vacuum level alignment, i.e., regime (ii) of the ICT model. It is worth to mention that the ionization potential of NTCDA is invariant with respect to the chosen substrates, which suggests no substrate-induced changes in ordering of the films. ${ }^{14}$

The calculated electron pinning energy $\mathrm{E}_{\mathrm{ICT}-}=4.53 \mathrm{eV}$ for a layer of flat lying NTCDA molecules in a herringbonelike structure similar to that used previously for PTCDA $(3,4,9,10 \text {-perylene-tetra-carboxylic-dianhydride })^{15}$ is in fair agreement with the experimental value. $\mathrm{E}_{\mathrm{ICT}-}$ includes a contribution of $0.12 \mathrm{eV}$ corresponding to a structural relaxation of the NTCDA molecule upon charging it with an electron. This relaxation energy is approximately twice that found for PTCDA, reflecting the smaller size of the NTCDA molecule. The calculated hole pinning energy $\mathrm{E}_{\mathrm{ICT}+}$ for an NTCDA layer in the same geometry is $6.33 \mathrm{eV}$, which lies outside of the range of substrate work functions used in this study.

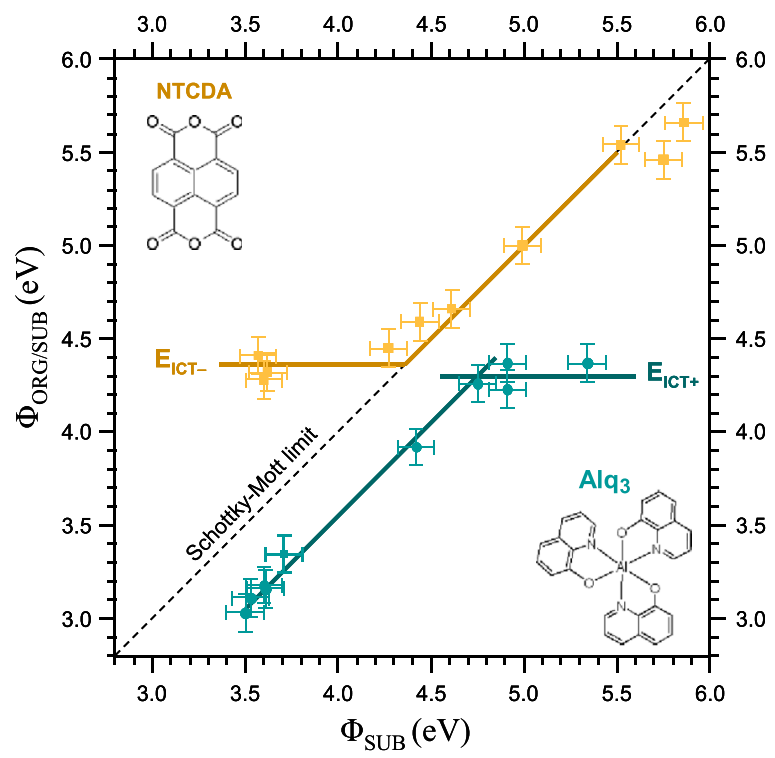

FIG. 1. Work function $\Phi_{\mathrm{ORG} / \mathrm{SuB}}$ of NTCDA (yellow) and Alq3 (teal) layers as function of the work function of the substrate $\Phi_{\mathrm{SUB}}$. The lines are added as a guide for the eye. The dashed line indicated the Schottky-Mott limit of vacuum level alignment.
For $\mathrm{Alq}_{3}$, the situation is more complex. $\mathrm{Alq}_{3}$ films formed by vacuum deposition under dark conditions may feature a phenomenon named giant surface potential. ${ }^{16}$ This effect stems from an alignment of the dipoles of the $\mathrm{Alq}_{3}$ molecules in the film, which builds up a large effective dipole in the film, and a corresponding electric field. The film dipole is not stable; it is sensitive to the UV light from the measurement, and it is permanently removed by extended irradiation. In the process of investigating the energy level alignment behavior of $\mathrm{Alq}_{3}$ care must hence be taken to first reach equilibrium, where the dipole alignment causing the giant surface potential is no longer present in the films.

From the $\mathrm{Alq}_{3}$ data in Figure 1, it is clear that the overall behavior of $\mathrm{Alq}_{3}$ follows the ICT model. For substrate work functions $\Phi_{\mathrm{SUB}}>\sim 4.7 \mathrm{eV}$, we observe Fermi level pinning, whereas for $\Phi_{\mathrm{SUB}}<\sim 4.7 \mathrm{eV}, \Phi_{\mathrm{ORG} / \mathrm{SUB}}$ follows the substrate work function. However, there is a displacement ( $\delta$ in Figure 1) in $\Phi_{\mathrm{ORG} / \mathrm{SUB}}$ of $\sim 0.4 \mathrm{eV}$ away from the SchottkyMott limit of vacuum level alignment. We attribute this displacement to a (partial) ordering of the $\mathrm{Alq}_{3}$ molecules at the interface with the substrate. Ordering (or partially ordering) the $\mathrm{Alq}_{3}$ molecules at the interface gives rise to an effective interface dipole, which leads to a potential step $\delta$ at the interface. The basic idea is illustrated in Figures 2 and 3. The displacement observed in Figure 1 is such that it effectively decreases the work function of the substrate. This indicates that the component of the effective dipole along the normal to the molecular layer is pointing away from the substrate. ${ }^{17}$ For substrate work functions $\Phi_{\mathrm{SUB}}>\sim 4.7 \mathrm{eV}$, the Fermi level pinning regime in Figure 1 gives a pinning value $\mathrm{E}_{\mathrm{ICT}+}=4.3 \mathrm{eV}$. Ordering the $\mathrm{Alq}_{3}$ molecules in a layer makes the vacuum level left $V L_{\mathrm{L}}$ and right $V L_{\mathrm{R}}$ of the layer different, the difference being the interface potential step $\delta$, see Figures 1 and 3. The substrate's vacuum level lines up

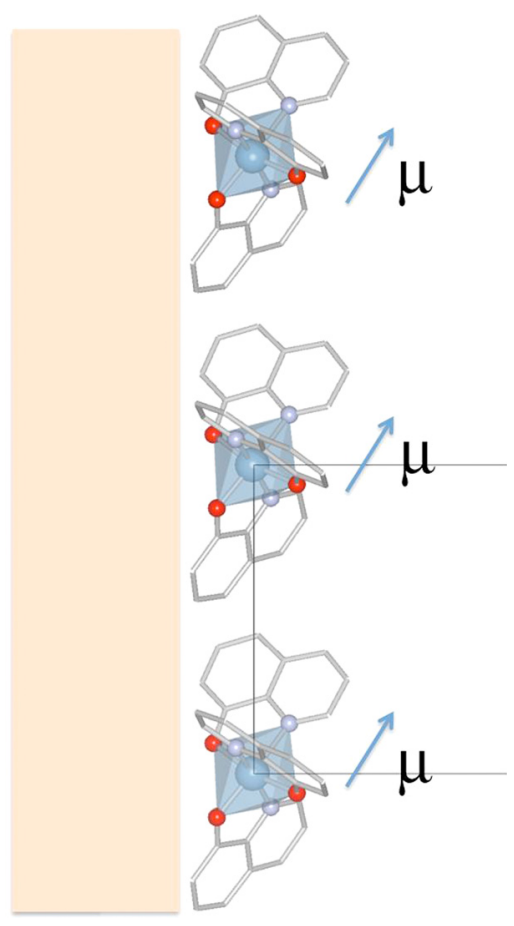

FIG. 2. An idealized structure of an ordered layer of (meridional) $\mathrm{Alq}_{3}$ molecules. The arrows indicate the molecular dipole moments. 


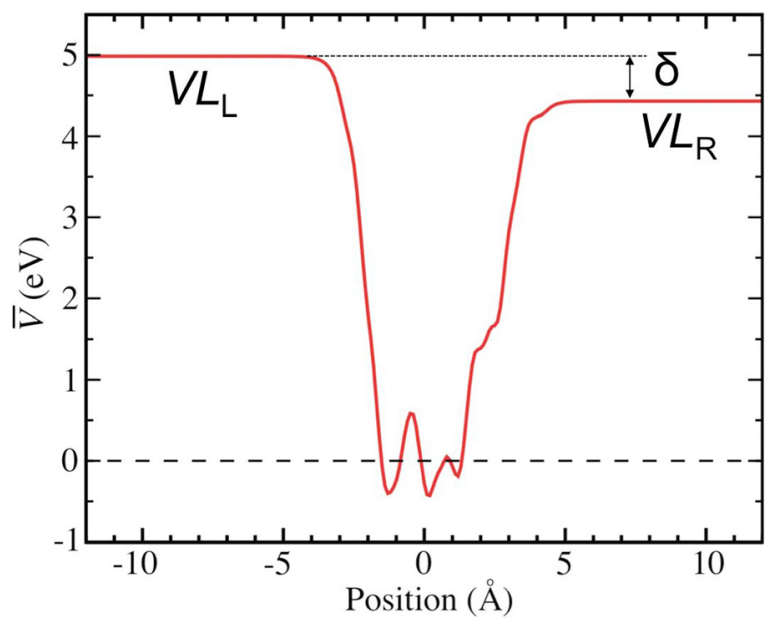

FIG. 3. The calculated plane-averaged electrostatic potential plotted along the normal to a free-standing $\mathrm{Alq}_{3}$ layer in the structure of Figure 2. The zero of the potential is placed at the calculated $\mathrm{E}_{\mathrm{ICT},+}$ level; $V L_{\mathrm{L}, \mathrm{R}}$ mark the vacuum potentials left and right of the layer, and $\delta=V L_{\mathrm{L}}-V L_{\mathrm{R}}$ marks the potential step induced by the layer.

with $V L_{\mathrm{L}}$, whereas for electrons extracted on the $\mathrm{Alq}_{3}$ side, the vacuum level is given by $V L_{\mathrm{R}}$.

A calculation on an ordered layer of $\mathrm{Alq}_{3}$ molecules in their meridional conformational form in the structure shown in Figure 2 gives a hole pinning level $\mathrm{E}_{\mathrm{ICT}+}=4.39 \mathrm{eV}$ and a potential step $\delta=0.56 \mathrm{eV}$, see Figure 3. The calculated molecular relaxation energy is $0.04 \mathrm{eV}$. The corresponding electron pinning level in this structure is $\mathrm{E}_{\mathrm{ICT}-}=2.65 \mathrm{eV}$, which would only be noticeable for substrates with a work function $\Phi_{\mathrm{SUB}}<\mathrm{E}_{\mathrm{ICT}-}+\delta$. The structure shown in Figure 2 is similar to that of a perfect $\mathrm{Alq}_{3}$ layer adsorbed on a clean metal substrate. ${ }^{18}$ It grasps the principal physical idea, but a calculation of the exact ordering of $\mathrm{Alq}_{3}$ molecules on substrates such as they are used in experiment is of course outside the scope of the present paper. The value of the pinning level is affected by the orientation and the packing of the $\mathrm{Alq}_{3}$ molecules within the layer. As an example, in the $\beta$-crystal structure of (meridional) $\mathrm{Alq}_{3}$, the molecular dipoles are locally ordered anti-parallel, ${ }^{19}$ which leads to low index crystal surfaces without a net effective dipole. For layers in (100), (111), and (210) orientations we find pinning levels in the range of $4.5-4.7 \mathrm{eV}$. A parallel ordering of molecular dipoles pointing away from the substrate, as in the structure of Figure 2, thus decreases the value of the pinning level.

Bilayer structures of NTCDA and $\mathrm{Alq}_{3}$ are made to test the ICT model for an organic multilayer featuring molecules with an intrinsic dipole. We use $\mathrm{AlO}_{\mathrm{x}}$ substrates with a work function of $3.5-3.6 \mathrm{eV}$. From the results of Figure 1, we can see that NTCDA has a pinning level for electrons $\mathrm{E}_{\mathrm{ICT}-\text {, NTCDA }} \sim 4.35 \mathrm{eV}$. Therefore, if NTCDA is deposited on $\mathrm{AlO}_{\mathrm{x}}$, electrons should be transferred from the substrate to the NTCDA layer, and the Fermi level should be pinned

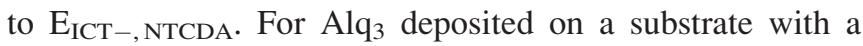
work function of $\sim 4.3 \mathrm{eV}$, such as an NTCDA layer on $\mathrm{AlO}_{\mathrm{x}}$, no further charge transfer is expected, as for $\mathrm{Alq}_{3}$, we are in the Schottky-Mott limit regime, see Figure 1.

Spectra on the sequentially formed $\mathrm{Alq}_{3} / \mathrm{NTCDA} / \mathrm{AlO}_{\mathrm{x}}$ system are shown in Figure 4. We see that the resulting work function upon NTCDA film formation on $\mathrm{AlO}_{\mathrm{x}}$ is $4.3 \mathrm{eV}$, in

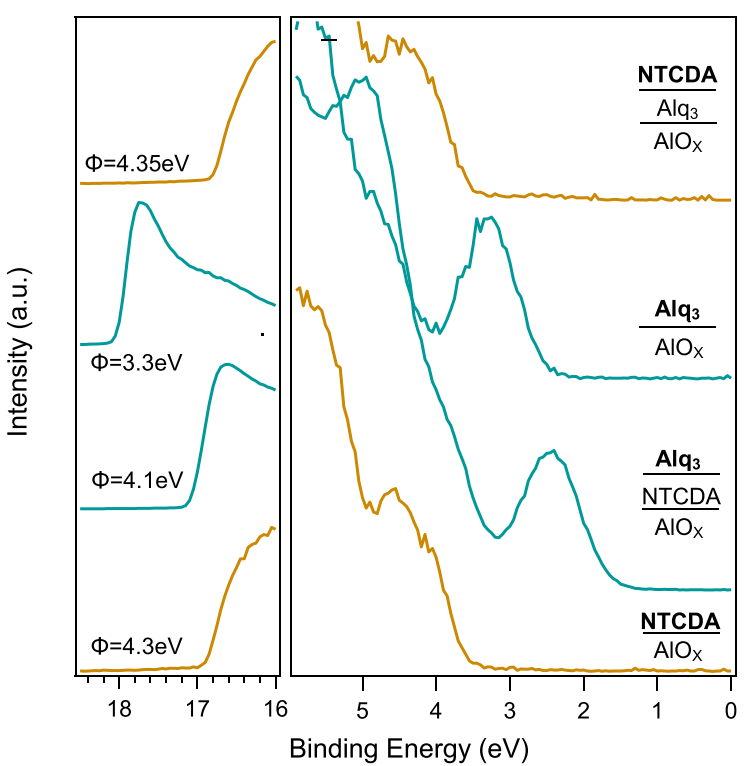

FIG. 4. UPS spectra of NTCDA and $\mathrm{Alq}_{3}$ films deposited in various configurations on $\mathrm{AlO}_{\mathrm{x}}$ substrate.

accordance with the ICT model predictions given the accuracy of the measurement technique. Depositing an $\mathrm{Alq}_{3}$ overlayer (and waiting for equilibrium) yields a small interface potential step of $0.2 \mathrm{eV}$. We attribute this step to a partially ordered $\mathrm{Alq}_{3}$ layer at the $\mathrm{Alq}_{3} / \mathrm{NTCDA}$ interface. The potential step is smaller than the $0.4 \mathrm{eV}$ obtained for $\mathrm{Alq}_{3}$ deposition on inorganic substrates. The UPS spectra on $\mathrm{Alq}_{3}$ deposited on NTCDA show a slight broadening of the Alq3 frontier peak as compared to $\mathrm{Alq}_{3} / \mathrm{AlO}_{\mathrm{x}}$ case. This is consistent with a less well-ordered $\mathrm{Alq}_{3}$ layer on a rougher NTCDA surface, which decreases the net collective dipole of the $\mathrm{Alq}_{3}$ molecules.

For $\mathrm{Alq}_{3}$ and the NTCDA layers deposited in the opposite sequential order onto $\mathrm{AlO}_{\mathrm{x}}$, i.e., $\mathrm{NTCDA} / \mathrm{Alq}_{3} / \mathrm{AlO}_{\mathrm{x}}$, there is no charge transfer between $\mathrm{Alq}_{3}$ and $\mathrm{AlO}_{\mathrm{x}}$, as for $\mathrm{Alq}_{3}$, we are still in the Schottky-Mott limit regime. The potential step of $0.3 \mathrm{eV}$ observed at the $\mathrm{AlO}_{\mathrm{x}} / \mathrm{Alq}_{3}$ interface is then again consistent with a (partial) ordering of $\mathrm{Alq}_{3}$ dipoles at the interface. Most remarkable, after NTCDA is deposited on $\mathrm{Alq}_{3}$, the work function changes considerably, i.e., from $3.3 \mathrm{eV}$ to $4.35 \mathrm{eV}$. The latter value corresponds to the pinning level $\mathrm{E}_{\mathrm{ICT}-\text {, NTCDA. }}$

The dependence of the results on $\mathrm{Alq}_{3} / \mathrm{NTCDA}$ on the deposition order can be reconciled with the ICT model. At the $\mathrm{Alq}_{3} / \mathrm{NTCDA}$ interface, the Fermi level is pinned to the level $\mathrm{E}_{\mathrm{ICT}-, \mathrm{NTCDA}}$. The difference between $\mathrm{E}_{\mathrm{ICT}-, \text { NTCDA }}$ $(4.35 \mathrm{eV})$ and $\mathrm{E}_{\mathrm{ICT}+\text {, Alq } 3}(4.3 \mathrm{eV})$ yields a $0.05 \mathrm{eV}$ dipole at the interface. This means that an additional potential increase of $4.3-3.3=1.0 \mathrm{eV}$ must occur to achieve equilibrium, and as a consequence, electrons are transferred across the $\mathrm{Alq}_{3}$ layer from the $\mathrm{AlO}_{\mathrm{x}}$ substrate to the NTCDA layer. This situation is reminiscent to the other donor-acceptor system, composed of tetrathiafulvalene (TTF) and tetracyanoquinodimethane (TCNQ), for which the charge equilibration occurs with the charge transfer from the substrate across the TTF film to TCNQ overlayer. ${ }^{6}$

In summary, the results for the single layer films as well for the bilayer structure of NTCDA and $\mathrm{Alq}_{3}$ agree well with the ICT model. The calculated values for the pinning levels 
and dipoles are in good agreement with the experimental data. The effect of a strong intrinsic molecular dipole on energy level alignment has been explored and shown to introduce a vacuum level shift in the absence of charge transfer across the interface. The small but significant spread in the measured dipole energy is attributed to variations in the $\mathrm{Alq}_{3}$ intermolecular order at the different interfaces, with rougher surfaces likely leading to a smaller dipole. The presence of an intrinsic dipole also shifts the onset of Fermi level pinning, as it increases (or reduces depending on direction) the energy for spontaneous charge transfer across the interface.

We acknowledge funding from the European Community's Framework Programme under Grant No. FP7NMP-228424 of the MINOTOR project as well as a project grant from the Swedish Energy Agency, STEM.

${ }^{1}$ H. Vazquez, F. Flores, and A. Kahn, Org. Electron. 8, 241 (2007); H. Vázquez, W. Gao, F. Flores, and A. Kahn, Phys. Rev. B 71, 041306R (2005); H. Vázquez, R. Oszwaldowski, P. Pou, J. Ortega, R. Pérez, F. Flores, and A. Kahn, Europhys. Lett. 65, 802 (2004).

${ }^{2}$ C. Tengstedt, W. Osikowicz, W. R. Salaneck, I. D. Parker, C. H. Hsu, and M. Fahlman, Appl. Phys. Lett. 88(5), 053502 (2006).

${ }^{3}$ M. Fahlman, A. Crispin, X. Crispin, S. K. M. Henze, M. P. de Jong, W. Osikowicz, C. Tengstedt, and W. R. Salaneck, J. Phys.: Condens. Matter 19, 183202 (2007).

${ }^{4}$ S. Braun, W. R. Salaneck, and M. Fahlman, Adv. Mater. 21(14), 1450 (2009).

${ }^{5}$ S. Braun, M. P. de Jong, W. Osikowicz, and W. R. Salaneck, Appl. Phys. Lett. 91, 202108 (2007).
${ }^{6}$ S. Braun, X. Liu, W. R. Salaneck, and M. Fahlman, Org. Electron. 11(2), 212 (2010).

${ }^{7}$ G. Brocks, D. Çakır, M. Bokdam, M. P. de Jong, and M. Fahlman, Org. Electron. 13, 1793 (2012)

${ }^{8}$ H. Aarnio, P. Sehati, S. Braun, M. Nyman, M. P. de Jong, M. Fahlman, and R. Österbacka, Adv. Energy Mater. 1, 792 (2011).

${ }^{9}$ M. Bokdam, D. Çakır, and G. Brocks, Appl. Phys. Lett. 98, 113303 (2011).

${ }^{10}$ W. Osikowicz, M. P. de Jong, and W. R. Salaneck, Adv. Mater. 19, 4213 (2007).

${ }^{11}$ V. Gohri, S. Hofmann, S. Reineke, T. Rosenow, M. Thomschke, M. Levichkova, B. Lüssem, and K. Leo, Org. Electron. 12(12), 2126 (2011); M. Kim, Y. S. Lee, Y. C. Kim, M. S. Choi, and J. Y. Lee, Synth. Met. 161, 2318 (2011); B. E. Lassiter, G. Wei, S. Wang, J. D. Zimmerman, V. V. Diev, M. E. Thompson, and S. R. Forrest, Appl. Phys. Lett. 98(24), 243307 (2011); S. Tanida, K. Noda, H. Kawabata, and K. Matsushige, Thin Solid Films 518(2), 571 (2009).

${ }^{12}$ A. Curioni, M. Boero, and W. Andreoni, Chem. Phys. Lett. 294, 263 (1998).

${ }^{13}$ D. Çakir, M. Bokdam, M. P. de Jong, M. Fahlman, and G. Brocks, Appl. Phys. Lett. 100, 203302 (2012).

${ }^{14}$ S. Duhm, G. Heimel, I. Salzmann, H. Glowatzki, R. L. Johnson, A. Vollmer, J. P. Rabe, and N. Koch, Nature Mater. 7, 326 (2008); W. Chen, H. Huang, S. Chen, Y. L. Huang, X. Y. Gao, and A. T. S. Wee, Chem. Mater. 20, 7017 (2008).

${ }^{15}$ P. C. Rusu, G. Giovannetti, C. Weijtens, R. Coehoorn, and G. Brocks, J. Phys. Chem. C 113, 9974 (2009); Phys. Rev. B 81, 125403 (2010).

${ }^{16}$ E. Ito, Y. Washizu, N. Hayashi, H. Ishii, N. Matsuie, K. Tsuboi, Y. Ouchi, Y. Harima, K. Yamashita, and K. Seki, J. Appl. Phys. 92(12), 7306 (2002).

${ }^{17}$ Y. Okabayashi, E. Ito, T. Isoshima, and M. Hara, Appl. Phys. Exp. 5, 055601 (2012).

${ }^{18}$ S. Yanagisawa and Y. Morikawa, J. Phys.: Condens. Matter 21, 064247 (2009).

${ }^{19}$ M. Brinkmann, G. Gadret, M. Muccini, C. Taliani, N. Masciocchi, and A. Sironi, J. Am. Chem. Soc. 122, 5147 (2000). 